\title{
The effect of caffeine on working memory load--related brain activation in middle--aged males
}

Citation for published version (APA):

Klaassen, E. B., De Groot, R., Evers, E. A. T., Snel, J., Veerman, E. C. I., Ligtenberg, A. J. M., Jolles, J., \& Veltman, D. J. (2013). The effect of caffeine on working memory load--related brain activation in middle--aged males. Neuropharmacology, 64, 160-167. https://doi.org/10.1016/j.neuropharm.2012.06.026

DOI:

10.1016/j.neuropharm.2012.06.026

Document status and date:

Published: 01/01/2013

Document Version:

Peer reviewed version

Document license:

CC BY-NC-ND

Please check the document version of this publication:

- A submitted manuscript is the version of the article upon submission and before peer-review. There can be important differences between the submitted version and the official published version of record. People interested in the research are advised to contact the author for the final version of the publication, or visit the DOI to the publisher's website.

- The final author version and the galley proof are versions of the publication after peer review.

- The final published version features the final layout of the paper including the volume, issue and page numbers.

Link to publication

\section{General rights}

Copyright and moral rights for the publications made accessible in the public portal are retained by the authors and/or other copyright owners and it is a condition of accessing publications that users recognise and abide by the legal requirements associated with these rights.

- Users may download and print one copy of any publication from the public portal for the purpose of private study or research.

- You may not further distribute the material or use it for any profit-making activity or commercial gain

- You may freely distribute the URL identifying the publication in the public portal.

If the publication is distributed under the terms of Article 25fa of the Dutch Copyright Act, indicated by the "Taverne" license above, please follow below link for the End User Agreement:

https://www.ou.nl/taverne-agreement

Take down policy

If you believe that this document breaches copyright please contact us at:

pure-support@ou.nl

providing details and we will investigate your claim.

Downloaded from https://research.ou.nl/ on date: 26 Apr. 2023 


\section{The effect of caffeine on working memory load-related brain activation in middle-aged males}

Elissa B. Klaassena,f, Renate H. M. de Groota,b,f, Elisabeth A. T. Eversc , Jan Sneld, Enno C.I. Veermane, Antoon J.M. Ligtenberge, Jelle Jolles ${ }^{\mathrm{f}}$, Dick J. Veltmang

a School for Mental Health and Neuroscience (MHeNS), Department of Psychiatry and Neuropsychology, Maastricht University, The Netherlands.

b Centre for Learning Sciences and Technologies (CELSTEC), Open University, The Netherlands. Email: Renate.deGroot@ou.nl

c Center for functional Magnetic Resonance Imaging, Department of Radiology, University of California San Diego (UCSD), San Diego, USA. Email: eevers@ucsd.edu

d Department of Psychology, Workgroup Brain and Cognition, University of Amsterdam, The Netherlands. Email: I.Snel@uva.nl

e Department of Oral Biochemistry, Academic Centre for Dentistry Amsterdam, The Netherlands. Email: e.veerman@acta.nl and a.ligtenberg@acta.nl

${ }^{\mathrm{f}}$ AZIRE Research Institute \& Faculty of Psychology and Education, VU University Amsterdam, The Netherlands. Email: j.jolles@psy.vu.nl

g Department of Psychiatry, VU University Medical Centre, \& Neuroscience Campus Amsterdam, The Netherlands. Email: DJ.Veltman@vumc.nl

Corresponding author: Elissa Klaassen

Educational Neuroscience, Centre of Brain and Learning, Vrije Universiteit Amsterdam, Van der Boechorststraat 1, 1081 BT Amsterdam, The Netherlands Tel.: +31 205988894

Email: e.b.klaassen@vu.nl / elissa.klaassen@maastrichtuniversity.nl 


\begin{abstract}
Caffeine is commonly consumed in an effort to enhance cognitive performance. However, little is known about the usefulness of caffeine with regard to memory enhancement, with previous studies showing inconsistent effects on memory performance. We aimed to determine the effect of caffeine on working memory (WM) load-related activation during encoding, maintenance and retrieval phases of a WM maintenance task using functional magnetic resonance imaging (fMRI). 20 healthy, male, habitual caffeine consumers aged 40 to 61 years were administered $100 \mathrm{mg}$ of caffeine in a double-blind placebo-controlled crossover design. Participants were scanned in a non-withdrawn state following a workday during which caffeinated products were consumed according to individual normal use (range $=145-595 \mathrm{mg}$ ). Acute caffeine administration was associated with increased load-related activation compared to placebo in the left and right dorsolateral prefrontal cortex during WM encoding, but decreased load-related activation in the left thalamus during WM maintenance. These findings are indicative of an effect of caffeine on the fronto-parietal network involved in the top-down cognitive control of WM processes during encoding and an effect on the prefrontal cortico-thalamic loop involved in the interaction between arousal and the top-down control of attention during maintenance. Therefore, the effects of caffeine on WM may be attributed to both a direct effect of caffeine on WM processes, as well as an indirect effect on WM via arousal modulation. Behavioral and fMRI results were more consistent with a detrimental effect of caffeine on WM at higher levels of WM load, than caffeine-related WM enhancement.
\end{abstract}

Keywords: Caffeine; Working memory; fMRI; Dorsolateral prefrontal cortex; Thalamus 


\section{Introduction}

Caffeine (a methylxanthine) is one of the most widely consumed psychoactive substances in the world, with coffee generally accounting for the majority of dietary caffeine intake (Nehlig, 1999). As a result of its stimulant properties, caffeine is often consumed in an attempt to enhance performance and combat the detrimental effects of fatigue. However, although caffeine has consistently been shown to diminish tiredness, increase energy and improve mood, findings regarding its ability to enhance cognitive performance are more complex (Nehlig, 2010; Snel et al., 2004).

Relatively consistent effects have been found with regard to the enhancement of psychomotor function (e.g. speeded reaction time) and sustained attention following caffeine consumption. Effects on learning and memory performance, however, appear to be more variable. Variable effects may be attributed to the diversity of caffeine research protocols in terms of caffeine dosage, cognitive task type and the characteristics (e.g., age, gender and habitual caffeine consumption levels) and state (e.g., fatigue and arousal level and caffeine abstinence) of the participant. Fortunately, insight into the effects of psychoactive agents, such as caffeine, on cognitive processes (regardless of whether significant changes in performance are evident at the behavioural level) can be provided by functional magnetic resonance imaging (fMRI). In the present study, we used fMRI to investigate the effect of caffeine on working memory (WM).

To date, just one study has investigated the effect of caffeine on learning or memory using fMRI. Koppelstaetter et al. (2008) demonstrated increased WM load-related activation (N-back task, block design) following caffeine (compared to placebo) administration in prefrontal cortex areas (PFC) associated with executive and attentional functions (in the absence of a significant effect on WM performance). This finding was suggested to reflect a direct effect of caffeine on WM processes. Behavioural findings, on the other hand, are generally considered to indicate an indirect effect of caffeine on WM via changes in arousal state.

We aimed to extend on findings by Koppelstaetter et al. (2008) by investigating the effect of caffeine on WM load-related activation during encoding, maintenance and retrieval phases of a WM maintenance task (modified Sternberg task) using an event-related fMRI design. In contrast to 
Koppelstaetter et al. (2008), we tested participants following a day of habitual caffeine consumption, rather than requiring an extended period of caffeine abstinence prior to testing. The advantage of this approach is that the effects of caffeine are investigated in the context of each participant's usual levels of caffeine in the body, instead of in the context of a caffeine withdrawal state. This design addresses the criticism that the beneficial effects of caffeine in abstained habitual consumers may in fact reflect the removal of the negative effects of caffeine withdrawal (Field et al., 2003; Heatherley, 2011; James, 1994). Caffeine withdrawal is associated with headaches, fatigue, dysphoric mood changes and flu-like somatic symptoms (Ozsungur et al., 2009), as well as changes in cerebral blood flow (CBF), the effect of which will be elucidated further below in relation to fMRI. In line with Koppelstaetter et al. (2008), we examined the effects of a $100 \mathrm{mg}$ dose of caffeine (equivalent to $1-1.5$ cups of coffee) in male caffeine consumers (250 - $800 \mathrm{mg}$ per day).

Our study focused on middle-aged males aged 40 to 61 years. Cognitive performance, including WM, has already started to decline by middle age (e.g. Bopp and Verhaeghen, 2005; Myerson et al., 2003; Park et al., 2002), as have energetic resources (Salthouse, 1988). Nevertheless, middle-aged adults commonly still work fulltime in positions that demand a high level of cognitive performance. There is some indication that caffeine can exert a more pronounced enhancement of cognition in older individuals (Nehlig, 2010). Therefore, the possible memory enhancing effects of caffeine are of particular interest in this age group; middle-aged adults may stand to benefit more from caffeine in terms of work-related performance enhancement than young adults (or retired old adults in whom daily cognitive demands are probably reduced). Since the memory enhancing effects of caffeine may be more evident when participants are in a suboptimal state (Nehlig, 2010), we tested participants immediately after a full day of work, when work-related fatigue levels are high.

When investigating the effect of caffeine on the blood oxygen level-dependent (BOLD) response utilised by fMRI as an indirect measure of neural activity, it is important to recognise that changes in the BOLD response can arise from both neural and cerebrovascular mechanisms (for a review see Koppelstaetter et al., 2010; Laurienti et al., 2003). Caffeine, a non-selective adenosine receptor antagonist (Pelligrino et al., 2010), elicits: 1) neurostimulant effects, primarily via $A_{1}$ 
receptors and the dopamine system (Ferre, 2008; Pelligrino et al., 2010); 2) cerebrovascular effects, primarily via $A_{2 A}$ and $A_{2 B}$ receptors located on blood vessels (Pelligrino et al., 2010); and 3) arousal enhancing effects, via $A_{2 A}$ receptors and the histaminergic arousal system (Ferre, 2008); with tolerance thought to arise as the brain regulates its population of adenosine receptors to reach a new state of equilibrium in response to levels of caffeine chronically present in the body (Jacobson et al., 1996; Ralevic and Burnstock, 1998; Sousa et al., 2011). Studies have shown that administration of 200 - 250 mg of caffeine results in reduced CBF (Addicott et al., 2009; Field et al., 2003; Laurienti et al., 2003; Liau et al., 2008; Mulderink et al., 2002; Perthen et al., 2008), possibly accompanied by a reduction in the baseline BOLD signal (during simple motor and visual tasks) (Chen and Parrish, 2009b; Perthen et al., 2008) and changes in the magnitude (Griffeth et al., 2011; Laurienti et al., 2002) and temporal dynamics of the BOLD response (Liau et al., 2008; Liu et al., 2004; Rack-Gomer et al., 2009). Furthermore, increased CBF has been found during caffeine withdrawal (Addicott et al., 2009; Field et al., 2003). Therefore, findings from studies examining the effect of caffeine on brain activation in the context of caffeine withdrawal (compared to the withdrawal state as a control condition) may represent the resolution of withdrawal-related activation changes as well as changes relating to the effects of caffeine on CBF and cognition (Field et al., 2003). However, there is evidence that lower caffeine doses do not affect the amplitude or timing of the BOLD response (Chen and Parrish, 2009a) and have a smaller effect (Chen and Parrish, 2009a), or do not significantly affect (Kennedy and Haskell, 2011), CBF. Furthermore, caffeine-related CBF changes are smaller in non-abstained higher consuming habitual users (Addicott et al., 2009). Therefore, our decision to administer a lower caffeine dosage to non-abstained moderate to high habitual caffeine consumers was intended to minimise the potential cerebrovascular effects of caffeine administration and avoid caffeine withdrawal.

In summary, we investigated the effect of caffeine on WM load-related activation in middleaged, male habitual caffeine consumers. Participants were tested following a workday during which they consumed caffeine according to their own habitual regime. Based on findings by Koppelstaetter et al. (2008), we hypothesised that (although this dose may not significantly affect WM performance at 
the behavioural level) caffeine would increase WM load-related activation in the fronto-parietal network associated with the top-down control of attention and executive functions.

\section{Methods}

\subsection{Participants}

Twenty-one right-handed Dutch male participants (aged 40 - 61) were recruited via advertisements in local newspapers. Inclusion criteria specified a high level of education, fulltime employment in a white-collar profession (to ensure greater sample homogeneity with regard to cognitive demands throughout the workday and the resulting cognitive fatigue state) and consumption of 250 to $800 \mathrm{mg}$ of caffeine daily. Daily caffeine consumption estimates at screening were based on participants' self-reports, with the estimate in mg calculated according to a recent overview of caffeine-containing products in the Netherlands (Wendte et al., 2003). The most commonly consumed products were estimated as follows: one cup $(125 \mathrm{ml})$ of filter coffee $=85 \mathrm{mg}$ of caffeine, instant coffee $(125 \mathrm{ml})=60 \mathrm{mg}$, espresso $(50 \mathrm{ml})=65 \mathrm{mg}$, and black tea $(125 \mathrm{ml})=30 \mathrm{mg}$. Exclusion criteria were significant past or present physical or psychiatric illness, medication use, illicit substance or nicotine use, or MRI contraindications. Females were not included due to gender differences in caffeine consumption and metabolism (Carrillo and Benitez, 1996; Magkos and Kavouras, 2005). The study was approved by the medical ethical committee at Maastricht University academic hospital. Volunteers gave informed consent prior to their (paid) participation.

\subsection{Design}

The study employed a randomised double-blind placebo-controlled crossover design. Participants were tested once after drinking caffeinated coffee (caffeine condition) and once after decaffeinated coffee (placebo condition) (treatment order was counterbalanced). Test sessions took place after a full workday (starting between 1600 and $1800 \mathrm{~h}$ ), including the participant's habitual regime of caffeine consumption, with the second session scheduled on the same day of the week, at the same time, as the first session, with one week between sessions. Participants and experimenters were blind to the caffeine content of the coffee preparation. Moreover, participants were not informed prior to testing that they would be receiving either caffeinated or decaffeinated coffee; they were simply 
asked to drink a cup of coffee. This addressed the role of expectations held by caffeine consumers regarding the effects of coffee on cognitive performance and mood (Dawkins et al., 2011; Kirsch and Weixel, 1988), as expectancy effects were kept constant across the placebo and caffeine conditions. Participants were informed about the caffeine and placebo conditions during debriefing.

\subsection{Caffeine manipulation}

In the caffeine condition, $100 \mathrm{mg}$ of caffeine (anhydrous) was mixed into a preparation of decaffeinated instant coffee. This dose was chosen to reflect caffeine dosages commonly consumed by habitual coffee drinkers and to minimise physiological side effects such as heart rate, blood pressure, CBF and BOLD response changes (Chen and Parrish, 2009a; Kennedy and Haskell, 2011; Koppelstaetter et al., 2008). The placebo treatment was a cup of decaffeinated instant coffee. Although decaffeinated coffee is not caffeine free (it contains $<3 \mathrm{mg}$ caffeine), it is considered an effective placebo as behavioural effects are not apparent at this dose and effects on the BOLD response do not differ between decaffeinated coffee and water (Bendlin et al., 2007).

\subsection{Subjective rating scales}

The Karolinska sleepiness scale (KSS) was administered as a measure of subjective feelings of sleepiness/alertness ( 1 = very alert, 9 = very sleepy) (Akerstedt and Gillberg, 1990), with demonstrated sensitivity to caffeine effects (Mednick et al., 2008). The Dutch short visual analogue scale (VAS) version of the Profile of Mood States (POMS) (Wald and Mellenbergh, 1990) was administered; the fatigue ( 6 items: $0=$ low fatigue, $100=$ high fatigue) and vigour ( 5 items: $0=$ low vigour, $100=$ high vigour) subscales are recommended measures of the mood of energy and fatigue in investigations that are short in duration (O'Connor, 2006) and the vigour scale has shown sensitivity to caffeine effects (Childs and de Wit, 2008; Herz, 1999). Finally, several additional VAS items (0 = 'not at all', 100 = 'extremely') were administered (post-coffee only) targeting caffeine-related experiences (Liguori et al., 1997) including: "Have you felt any caffeine effects?" (also used as an evaluation of blinding effectiveness), “Have you felt any good effects?", and "Have you felt any bad effects?”.

\subsection{Salivary caffeine concentrations}


Saliva samples were collected pre-coffee (time 0) and 25 min (time 1) and 90 min (time 2) post-coffee using Salivettes (Sarstedt, the Netherlands). Saliva sampling is considered a good method for frequent measurement of caffeine pharmacokinetics (Newton et al., 1981; Suzuki et al., 1989), with a linear relationship reported between caffeine concentrations in saliva and plasma, and salivary caffeine levels reported to be around $80 \%$ of plasma levels (Perera et al., 2011; Zylber-Katz et al., 1984). Collection, storage and analysis procedures are supplied as Supplementary Material.

\section{6 fMRI visual control task}

In the visual control task, alternating blocks (12 s) of stationary dots, moving dots or no dots were presented (Goebel et al., 1998). Participants were instructed to look at a fixation-cross presented in the centre of the screen throughout all blocks. This task was administered to assess whether caffeine exerted a global effect on the BOLD response (Iannetti and Wise, 2007).

\section{7 fMRI working memory task}

Participants performed a parametric version of the letter Sternberg task $(3,4,5$ or 6 letter strings). The task consisted of 144 trials (36 for each letter string length) organised in a fast eventrelated design and presented in a fixed pseudorandom order (stimulus letters, but not trial order, varied per task version). Experimental trials consisted of encoding, maintenance and retrieval phases. Participants were instructed to memorise the letter string (lower case consonants) presented on the screen (for $4 \mathrm{~s}$ ) during the encoding phase. The maintenance phase followed, consisting of a fixation cross in the centre of the screen (for a jittered interval of 3 to 6 s). Finally, in the retrieval phase, participants responded to a probe letter by pressing a button to indicate whether the probe letter was part of the previous letter string (right button press) or not (left button press). Trials were separated by a 3 s pause (during which time three asterisks were shown in the centre of the screen). In addition to jittering, partial trials were also included to reduce the problem of multicollinearity arising from the delayed nature of the BOLD response inherent to tasks, such as the Sternberg task, with a fixed stimulus event order (Dale, 1999; Miezin et al., 2000; Ollinger et al., 2001a; Ollinger et al., 2001b; Serences, 2004). The partial trial technique allows the unique estimation of the different trial phases and has been shown to be more effective than jittering or slow event-related designs for the detection of cue-related activity in the prefrontal cortex (PFC) (Goghari and MacDonald, 2008). 50\% of all trials 
consisted of the encoding and maintenance phases only and a further $17 \%$ consisted of the encoding phase only. Participants were therefore not required to respond to these incomplete trials, but were required to encode and maintain, or encode only, respectively. The task was administered in three blocks of approximately 11 min each and 48 trials per block.

\subsection{Procedure}

Firstly, participants completed a diary recording food and beverage type, quantity and time of consumption during one workday (the same workday as the test sessions were subsequently held on). The diary provided a more precise estimate of the amount of caffeine consumed by each participant during the workday. Furthermore, the diary ensured consistency with regard to the concentration of caffeine in the body at the start of the two test sessions; each participant's pattern of caffeine consumption reported in the diary was used as a schedule for their caffeine consumption on both test days, with the exception that caffeine sources normally consumed during the $2 \mathrm{~h}$ prior to the start of the test sessions were removed. This abstinence period of at least $2 \mathrm{~h}$ (exact times depended on individual habits) ensured that caffeine levels were relatively low at the beginning of the test sessions (but that participants were not in caffeine withdrawal) and that subsequent coffee administration did not result in excessive levels of caffeine in the body. Participants confirmed compliance to their caffeine schedule by recording actual consumption times throughout each test day.

During a training session, participants practiced the fMRI tasks in a dummy scanner to minimise practice effects during the test sessions and to become familiarised with the scanning environment. During the test sessions, participants filled out pre-coffee subjective rating scales and provided a baseline (time 0 ) saliva sample before drinking the coffee preparation and entering the MRI scanner. The scanning session began with a structural scan (10 min after coffee consumption), followed by saliva sample collection (time 1), the visual control task (30 min after coffee), and the WM task. Therefore, at the time of the first functional scan, the majority of the caffeine dose was expected to be absorbed and distributed in the brain (Liguori et al., 1997). After exiting the scanner, participants completed the post-coffee subjective rating scales and provided another saliva sample (time 2).

\subsection{MRI data acquisition}


Images were acquired in a 3 Tesla head scanner (Siemens MAGNETOM Allegra, Siemens Medical systems, Erlangen, Germany) with an 8 channel head coil. During each of the three WM task blocks, approximately 330 Echo-planar imaging (EPI) scans were made (TR $=2000 \mathrm{~ms}, \mathrm{TE}=30 \mathrm{~ms}$, number of slices $=32$, image matrix $=64 \times 64$, voxel size $=3.5 \times 3.5 \times 3.5 \mathrm{~mm}$ ) of the cortex. During the visual control task, approximately 110 EPI scans were made $(\mathrm{TR}=2000 \mathrm{~ms}$, TE $=30 \mathrm{~ms}$, number of slices $=28$, image matrix $=128 \times 128$, voxel size $=2 \times 2 \times 2 \mathrm{~mm}$ ) over the visual cortex. A T1 weighted anatomical (ADNI) scan was also acquired for anatomical reference and coregistration of the two test sessions (image matrix $=256 \times 256$, number of slices $=192$, voxel size $=1 \times 1 \times 1 \mathrm{~mm}$ ).

\subsection{MRI data analysis}

SPM8 (Statistical Parametric Mapping: Wellcome Trust Centre for Neuroimaging, Institute of Neurology, University College London) was used to preprocess the fMRI scans. Preprocessing steps included: slice time correction, realign and unwarp, coregistration (session 2 scans were coregistered to session 1 scans), spatial normalisation (MNI space using individual spatial normalisation parameters obtained during structural image segmentation), and smoothing (FWHM $6 \mathrm{~mm}$ ).

Visual control task: Data were analysed in the context of the general linear model, using boxcar regressors convolved with the canonical hemodynamic response to model experimental trial blocks. Additionally, we extracted the average fMRI time series from white matter and cerebrospinal fluid (CSF) using each participants segmented anatomical scan in the REST toolbox (Song et al., 2011) and added these as regressors to the model as a measure to correct for possible changes in physiological noise resulting from caffeine administration. Individual activation contrasts comparing activation during moving dot blocks to stationary dot blocks were entered into a second level paired-samples ttest to compare visual cortex activation in the placebo and caffeine conditions. Effects were tested at $p$ (uncorrected) <.001, with a voxel threshold of 5 voxels, small volume corrected to restrict the search for activation differences to the visual cortex.

Working memory task: Data were analysed in the context of the general linear model, using boxcar regressors convolved with the canonical hemodynamic response to model activation during each event type. Encoding events were modelled as $4 \mathrm{~s}$ blocks, maintenance events as blocks with duration dependent on jittering time and retrieval events as blocks with duration dependent on 
response time. Additionally, we again added the average fMRI time series from white matter and CSF as regressors in the model.

Individual activation contrasts were created by modelling activation to the WM load levels ( 3 , 4, 5, 6 letters) during encoding, maintenance and retrieval using a parametric load contrast (-2 - 1112$)$. Contrasts were entered into three second-level Full Factorial models to assess the effects of caffeine condition on load-related activation in each of the task phases. The effect of caffeine condition on whole-brain activation was examined at $p$ (uncorrected) $<.001$ with a cluster threshold $>5$ voxels (masked inclusively with task-related activation to restrict the search for activation differences to those areas showing an effect of WM load).

Region of interest (ROI) analysis was used to examine the effect of caffeine (at $p<.005$ ) within select fronto-parietal areas consistently modulated by WM load (as well as within any additional areas showing an effect of caffeine on activation at the whole-brain level) using the MarsBaR toolbox for SPM (Brett et al., 2002). ROIs where constructed by creating a sphere (10 mm radius) around peak load-related activation coordinates obtained from a previous study using the same task in a similar population of 30 males aged $25-60$, and then masking this sphere with a grey matter mask. ROIs included the bilateral dorsolateral prefrontal cortex (DLPFC) (MNI coordinates $[x=(-) 39, y=42, z=$ 30]; Brodmann area [BA] 46), precentral gyrus (coordinates [ $\mathrm{x}=(-) 51, \mathrm{y}=3, \mathrm{z}=42$ ]; BA 6), superior parietal (coordinates $[\mathrm{x}=(-) 24, \mathrm{y}=-66, \mathrm{z}=54]$; BA 7) and inferior parietal cortex (coordinates $[\mathrm{x}=(-$ J51, $y=-45, z=48]$; BA 40). Additionally, we used the MarsBaR toolbox to extract the fitted event time course from the ROIs showing a significant effect of caffeine to determine whether caffeine affected the temporal dynamics (time to peak) of the BOLD response underlying activation differences.

\subsection{Salivary caffeine, subjective ratings and working memory performance data analysis}

Analyses were carried out using PASWStatistics (version 18.0). Repeated measures analysis of variance (ANOVA) were used to examine the effect of caffeine condition and time on caffeine concentration in saliva and subjective ratings on the KSS and the POMS subscales, and the effect of caffeine condition and WM load on performance accuracy and RT on the WM task.

\section{Results}


One participant was excluded for failing to follow their workday caffeine consumption schedule leaving 20 participants for the analysis (mean age $=49.2, \mathrm{SD}=5.6$, range $=40-61$ ). Data was missing from one participant on the visual control task. Missing salivary caffeine values, due to an inadequate amount of saliva for analysis, were replaced with the value from the other test session (for baseline values) or with the individual's average from the previous and following time points (for time 1 and time 2 values, also using a time 3 measurement, 120 min after coffee consumption, not otherwise included in the analyses).

\subsection{Caffeine consumption and salivary concentrations}

Estimates of caffeine consumption during the workday prior to the test session indicated an average consumption of $335 \mathrm{mg}(\mathrm{SD}=104 \mathrm{mg}$, range = $145-595)$. The mean amount of caffeine administered per kg of body weight was $1.22 \mathrm{mg} / \mathrm{kg}(\mathrm{SD}=0.17$, range $=0.89-1.47 \mathrm{mg} / \mathrm{kg})$.

The concentration of caffeine in saliva is shown in Figure 1. A main effect of caffeine condition $(F(1,19)=8.55, p=.009)$ and time point was found $(F(2,38)=7.87, p=.005)$, as well as a trend for an interaction between caffeine condition and time point $(F(2,38)=2.92, p=.069)$. Follow-up tests indicated that caffeine concentrations did not differ between conditions at baseline (time 0 ), but were significantly higher in the caffeine than the placebo condition at time $1(t(19)=2.93, p=.009)$ and this difference neared significance at time $2(t(19)=2.02, p=.058)$.

\subsection{Subjective ratings}

Ratings on each of the scales are shown in Table 1. Caffeine condition had no significant effect on ratings on the KSS or the POMS fatigue or vigour scales. A main effect of time point was found on each of the scales indicating increased sleepiness $(F(1,19)=32.64, p<.001)$ and fatigue $(F(1,19)=$ $34.28, p<.001)$, and decreased vigour $(F(1,19)=30.80, p<.001)$ after the scanning session compared to the pre-coffee baseline, regardless of caffeine condition. Scores also did not differ between caffeine and placebo conditions on the additional caffeine VAS items targeting caffeine-related experiences, indicating that participants had no awareness of caffeine condition.

\subsection{Visual control task}

Visual task-related activation in the placebo condition in response to stimulation with moving compared to stationary dots was found bilaterally throughout the primary and secondary visual areas 
(peak coordinates: e.g. $[x=-22, y=-90, z=28],[x=-6, y=-84, z=0],[x=8, y=-82, z=-2],[x=26, y=$ $-86, \mathrm{z}=16]$; $\mathrm{t}$-values $=26.74,25.28,21.52,21.07$; cluster size $=4373, \mathrm{BA} 17 / 18)$. No activation differences were found between placebo and caffeine conditions.

\subsection{Working memory task behavioural results}

Accuracy and RT on the task are shown in Figure 2. A main effect of WM load was found on performance accuracy $(F(3,57)=5.09, p=.006)$, reflecting a decrease in accuracy with increasing load. A trend for an interaction between the effect of caffeine condition and WM load on accuracy was also found $(F(3,57)=2.68, p=.058)$, with follow-up tests showing significantly lower accuracy on six letter trials in the caffeine than in the placebo condition $(t(20)=2.15, p=0.044)$. A main effect of WM load was found on $\operatorname{RT}(F(3,57)=20.40, p<.001)$, reflecting increased RT with increasing WM load $(F(1$, 19) $=12.70, p=.002$ ). However, caffeine condition did not significantly affect RT.

\subsection{Working memory task fMRI results}

Whole brain analysis: Load-related activation during encoding, maintenance and recognition in the placebo and caffeine condition is shown in Table 2. Load-related encoding activation was greater in the caffeine than the placebo condition in the right DLPFC (peak coordinates: $[x=36, y=36, z=39]$; $\mathrm{t}$-value $=4.79$; cluster size $=23 ; \mathrm{BA} 9 / 46)$. Load-related maintenance activation was greater in the placebo than the caffeine condition in the left thalamus (peak coordinates: $[x=-15, y=-9, z=6], t-$ value $=4.24$, cluster size $=8$ ). No load-related activation differences were found during retrieval.

Region of interest analysis: Activation in ROIs showing significant differences in load-related activation in the placebo and caffeine condition is shown in Figure 3. ROI analysis confirmed greater load-related encoding activation in the caffeine than the placebo condition in the right DLPFC $(t(19)=$ $2.84, p=.004)$ and additionally showed the same effect in the left DLPFC $(t(19)=3.00, p=.002)$. In the right DLPFC, load-related activation was evident in the placebo condition $(t(19)=2.32, p=.013)$, and further increased in the caffeine condition $(t(19)=4.88, p<.001)$. In the left DLPFC, activation in the placebo condition was not modulated by WM load $(t(19)=0.61, p=.727)$, but showed a load-related increase in the caffeine condition $(t(19)=1.79, p=.041)$.

Greater load-related maintenance activation in the placebo than in the caffeine condition in the left thalamus $(t(19)=2.80, p=.004)$ reflected a load-related increase in the placebo condition $(t(19)=$ 
$2.38, p=.011)$, but somewhat of a load-related decrease in activation in the caffeine condition $(t(19)=$ $1.58, p=.061$ ). No other activation differences between the placebo and caffeine condition were found. Furthermore, we found no indication of differences in the temporal dynamics of the BOLD response in the DLPFC and thalamus between the placebo and caffeine conditions.

Post-hoc correlations: To gain insight into the relationship between the effect of caffeine on load-related activation and WM performance, we correlated activation within the left DLPFC, right DLPFC and left thalamus ROIs with performance accuracy and RT. A significant positive correlation was found between load-related activation in the right DLPFC and performance accuracy in the caffeine condition $(t(19)=2.95, p=.004)$. We also examined the relationship between load-related activation in the three ROIs and the factors age, daily caffeine consumption level and caffeine dose in $\mathrm{mg} / \mathrm{kg}$. However, no significant correlations were found.

\section{Discussion}

We examined the effects of $100 \mathrm{mg}$ of caffeine (compared to placebo) on WM load-related activation in non-withdrawn male habitual caffeine users at the end of the workday (when workrelated fatigue levels were expected to be relatively high). The measurement of caffeine concentration in saliva confirmed that concentrations were significantly higher in the caffeine compared to the placebo condition. Functional MRI showed an effect of caffeine on WM load-related activation in the bilateral DLPFC during encoding and in the left thalamus during maintenance, whereas no effects were found during retrieval.

The increase in load-related activation in the DLPFC in the caffeine compared to the placebo condition is suggestive of a direct effect of caffeine on WM processes. This finding is somewhat consistent with the study by Koppelstaetter et al. (2008) in which caffeine also increased WM loadrelated activation in the PFC. Although, activation changes in that study were evident in the bilateral medial frontopolar cortex (BA 10), extending into the ACC. This difference in the areas modulated by caffeine may be attributed to one or all of several important differences between the two studies including the type of WM task, fMRI design, and participant age and abstinence state. Nevertheless, both studies point towards a direct effect of caffeine on the fronto-parietal WM network, with our study specifically indicating an effect on this network during WM encoding. 
Studies have consistently demonstrated that the DLPFC is a key area in the brain's response to varying levels of WM load and have suggested a primary role for this area in the top-down control of attention and WM (Toro et al., 2008). Thus, it may be expected that enhanced DLPFC activation would be indicative of the facilitation of top-down cognitive control, thereby leading to enhanced WM encoding and a higher level of performance. Indeed, we found a positive correlation between loadrelated activation in the right DLPFC and overall performance accuracy in the caffeine condition. However, performance actually tended to worsen at the higher WM load levels in the caffeine compared to the placebo condition; somewhat longer RTs were evident in response to five letters followed by a decrease in accuracy at six letters. These behavioural findings are consistent with the suggestion, based on previous behavioural studies, that although caffeine appears to facilitate performance at low levels of WM demand, it is associated with impaired performance at higher levels of demand (Anderson and Revelle, 1983; Loke, 1993; Nehlig, 2010). Therefore, we suggest that although greater load-related recruitment of the right DLPFC during encoding in the caffeine condition was associated with better performance, the evident detrimental effect of caffeine on performance at higher levels of WM load overall indicates that the effect of caffeine on other processes (such as arousal, discussed below) may have been more instrumental in determining WM performance.

Load-related activation in the maintenance phase of the task was greater in the placebo than in the caffeine condition in the left thalamus. Previous studies have shown a load-related increase in activation in the left thalamus during WM tasks (Altamura et al., 2007; Choo et al., 2005; Nyberg et al., 2009). Furthermore, a specific role for the thalamus during WM maintenance has been proposed by Ashby et al. (2005), in which activation during the delay interval is sustained by an excitatory prefrontal cortico-thalamic loop (in conjunction to the fronto-parietal excitatory loop). As such, the thalamus may be differentially activated in an effort to support the maintenance of information in the absence of input from external stimuli.

An earlier fMRI study also demonstrated decreased thalamic activation following caffeine administration (compared to a sleep deprivation condition) (Portas et al., 1998) and attributed this finding to the proposed role of the thalamus in arousal regulation (Schiff, 2008). Specifically, higher levels of thalamic activation are found in relation to low levels of arousal (Chee et al., 2006; Foucher et 
al., 2004). Hence, we suggest that lower arousal levels in the placebo condition necessitated greater thalamic activation to support WM maintenance, whereas higher arousal levels in the caffeine condition necessitated less support from the thalamus. In relation to our behavioural findings, it has been suggested that the caffeine-related increase in arousal state, in combination with increased arousal resulting from high task demands, may lead to over-arousal and a subsequent decline in performance (Nehlig, 2010). Indeed, Foucher et al. (2004) showed that the left thalamus is modulated by both endogenous arousal levels as well as arousal changes in response to task stimuli. Therefore, although thalamic activation was not directly correlated with WM performance, it may have reflected an indirect effect of caffeine on performance via changes in arousal state. Specifically, increased arousal levels in the caffeine condition may have contributed to poorer WM performance at high WM load levels in this condition. Overall, findings are suggestive of a modulatory effect of caffeine on activation in the prefrontal cortico-thalamic loop involved in the interaction between arousal and the top-down control of attention during maintenance.

A major strength of the present study is that participants were not tested in a state of withdrawal. Therefore, differences found between the placebo and caffeine conditions cannot be attributed to resolution of the withdrawal state. Furthermore, additional use of a caffeine dosage equivalent to 1-1.5 cups of coffee means that results are more indicative of effects of caffeine as it is consumed in daily life. Although, the administration of this low dose to caffeine-tolerant habitual consumers probably also accounts for the absence of a significant effect of caffeine on subjective fatigue, vigour and sleepiness ratings and the subjective experience of caffeine-related effects. Similarly, the lack of a significant effect of caffeine on task accuracy or RT is not unusual in studies using a low dose in non-abstained habitual consumers (Christopher et al., 2005; Heatherley et al., 2005), whereas significant effects are more consistently found in studies testing participants in a withdrawal state using higher doses. Nevertheless, there are other advantages to using this low dose including minimal physiological side effects and effective blinding. We note that, although there is no reason to expect differential caffeine expectancy effects in the placebo and caffeine conditions, as participants were simply asked to drink a cup of coffee in both conditions, we can not rule out the possibility that expectancy effects may have contributed to results on our outcome measures in both 
conditions. We also note that the use of decaffeinated coffee (with or without added caffeine) does not take into account the possible effects of other active compounds in coffee on mood or cognition (Cropley et al., 2012) (which may have obscured caffeine effects), or a possible synergistic relationship between the effects of these active compounds and caffeine.

However, interpretations of our fMRI results are subject to one primary caveat. Namely, that caffeine can influence the BOLD response via both neural and vascular pathways. However, minimal vascular effects were expected due to the administration of a low caffeine dose to habitual users. Results from the visual control task support the expected absence of a global effect of caffeine on the magnitude of BOLD response, as the BOLD response to visual stimulation did not differ significantly between the caffeine and placebo conditions. Furthermore, no differences were evident in the temporal dynamics of the BOLD response in areas showing a significant effect of caffeine. Moreover, as explained by Koppelstaetter et al. (2008), the regional specificity of the effects of caffeine on the BOLD response during the WM task argues against an explanation solely based on vascular effects, as $\mathrm{A}_{1}$ receptors responsible for the neuroexcitatory effects of caffeine are expressed throughout the cortex. Similarly, the specificity of caffeine effects to the particular WM task phases in the present study is again inconsistent with a global effect of caffeine on parameters of the BOLD response. Finally, the use of a parametric contrast avoids the direct comparison of the BOLD response itself between caffeine and placebo conditions. Nevertheless, we note that the present study would have been improved by the addition of a measure providing direct insight into vascular effects (such as arterial spin labelling). Furthermore, although we endeavoured to correct for any possible effects of increases in physiological noise resulting from caffeine administration by including the average CSF and WM time series as regressors in the fMRI model, ideally, future studies should include physiological measures such as heart and respiration rate in order to properly monitor and correct for possible physiological changes.

In conclusion, the present study provides evidence for an effect of 1-1.5 cups of coffee on loadrelated activation during WM encoding and maintenance in 40 to 61 year-old male habitual caffeine consumers. During WM encoding, caffeine modulated activation in the right DLPFC, indicating an effect on the top-down cognitive control of WM. During WM maintenance, caffeine modulated activation in the left thalamus, indicating an effect on arousal. Therefore, the effects of caffeine on WM may be 
attributed to both a direct effect of caffeine on WM processes, as well as an indirect effect via changes in arousal. This finding is consistent with both the neurostimulant and arousal enhancing effects on caffeine primarily via the dopamine (Ferre, 2008; Pelligrino et al., 2010) and histaminergic arousal (Ferre, 2008) systems respectively. However, behavioural and fMRI results were more consistent with a detrimental effect of caffeine on WM at higher levels of WM load, than caffeine-related WM enhancement.

\section{Acknowledgements}

This research was supported by Maastricht University. The authors would like to thank Funs Boots, Karolina Nelissen and the participants for their contribution. 
Figure 1. Caffeine concentration in saliva at baseline (time 0 ) and at each post-coffee measurement (indicated in minutes after coffee consumption) ${ }^{1}$.

${ }^{1}$ Error bars show standard error and * indicates a significant difference between placebo and caffeine conditions.

Figure 2. Percentage accuracy and reaction time to each level of working memory load $(3,4,5$, or 6 letters) on the fMRI working memory task in the placebo and caffeine conditions ${ }^{1}$.

${ }^{1}$ Error bars show standard error and * indicates a significant difference between placebo and caffeine condition

Figure 3. Working memory load-related activation (percentage signal change, with error bars showing standard error) in the dorsolateral prefrontal cortex (DLPFC) during encoding and the left thalamus during maintenance in the placebo and caffeine condition 


\section{References}

Addicott, M. A., Yang, L. L., Peiffer, A. M., Burnett, L. R., Burdette, J. H., Chen, M. Y., Hayasaka, S., Kraft, R. A., Maldjian, J. A., Laurienti, P. J., 2009. The effect of daily caffeine use on cerebral blood flow: How much caffeine can we tolerate? Hum. Brain Mapp. 30, 31023114.

Akerstedt, T., Gillberg, M., 1990. Subjective and objective sleepiness in the active individual. Int. J. Neurosci. 52, 29-37.

Altamura, M., Elvevag, B., Blasi, G., Bertolino, A., Callicott, J. H., Weinberger, D. R., Mattay, V. S., Goldberg, T. E., 2007. Dissociating the effects of Sternberg working memory demands in prefrontal cortex. Psychiat. Res. 154, 103-114.

Anderson, K., Revelle, W., 1983. The interactive effects of caffeine, impulsivity and task demands on visual search task. Pers. Indiv. Differ. 4, 127-132.

Ashby, F. G., Ell, S. W., Valentin, V. V., Casale, M. B., 2005. FROST: a distributed neurocomputational model of working memory maintenance. J. Cognitive Neurosci. 17, 1728-1743.

Bendlin, B. B., Trouard, T. P., Ryan, L., 2007. Caffeine attenuates practice effects in word stem completion as measured by fMRI BOLD signal. Hum. Brain. Mapp. 28, 654-662.

Bopp, K. L., Verhaeghen, P., 2005. Aging and verbal memory span: a meta-analysis. J. Gerontol. B-Psychol. 60, 223-233.

Brett, M., Anton, J., Valabreque, R., Poline, J., 2002. Region of interest analysis using an SPM toolbox [abstract] Presented at the 8th International Conference on Functional Mapping of the Human Brain, Sendai, Japan. Neuroimage 16, Available on CD-ROM.

Carrillo, J. A., Benitez, J., 1996. CYP1A2 activity, gender and smoking, as variables influencing the toxicity of caffeine. Br. J. Clin. Pharmaco. 41, 605-608.

Chee, M. W., Chuah, L. Y., Venkatraman, V., Chan, W. Y., Philip, P., Dinges, D. F., 2006. Functional imaging of working memory following normal sleep and after 24 and $35 \mathrm{~h}$ of sleep deprivation: Correlations of fronto-parietal activation with performance. Neuroimage 31, 419-428.

Chen, Y., Parrish, T. B., 2009a. Caffeine dose effect on activation-induced BOLD and CBF responses. Neuroimage 46, 577-583.

Chen, Y., Parrish, T. B., 2009b. Caffeine's effects on cerebrovascular reactivity and coupling between cerebral blood flow and oxygen metabolism. Neuroimage 44, 647-652.

Childs, E., de Wit, H., 2008. Enhanced mood and psychomotor performance by a caffeinecontaining energy capsule in fatigued individuals. Exp. Clin. Psychopharm. 16, 13-21.

Choo, W. C., Lee, W. W., Venkatraman, V., Sheu, F. S., Chee, M. W., 2005. Dissociation of cortical regions modulated by both working memory load and sleep deprivation and by sleep deprivation alone. Neuroimage 25, 579-587.

Christopher, G., Sutherland, D., Smith, A., 2005. Effects of caffeine in non-withdrawn volunteers. Hum. Psychopharmacol. Clin. Exp. 20, 47-53.

Cropley, V., Croft, R., Silber, B., Neale, C., Scholey, A., Stough, C., Schmitt, J., 2012. Does coffee enriched with chlorogenic acids improve mood and cognition after acute administration in healthy elderly? A pilot study. Psychopharmacology 219, 737-749.

Dale, A. M., 1999. Optimal experimental design for event-related fMRI. Hum. Brain Mapp. 8, 109114.

Dawkins, L., Shahzad, F. Z., Ahmed, S. S., Edmonds, C. J., 2011. Expectation of having consumed caffeine can improve performance and mood. Appetite 57, 597-600.

Ferre, S., 2008. An update on the mechanisms of the psychostimulant effects of caffeine. J. Neurochem. 105, 1067-1079.

Field, A. S., Laurienti, P. J., Yen, Y. F., Burdette, J. H., Moody, D. M., 2003. Dietary caffeine consumption and withdrawal: confounding variables in quantitative cerebral perfusion studies? Radiology 227, 129-135.

Foucher, J. R., Otzenberger, H., Gounot, D., 2004. Where arousal meets attention: a simultaneous fMRI and EEG recording study. Neuroimage 22, 688-697. 
Goebel, R., Khorram-Sefat, D., Muckli, L., Hacker, H., Singer, W., 1998. The constructive nature of vision: direct evidence from functional magnetic resonance imaging studies of apparent motion and motion imagery. Eur. J. Neurosci. 10, 1563-1573.

Goghari, V. M., MacDonald, A. W., 3rd, 2008. Effects of varying the experimental design of a cognitive control paradigm on behavioral and functional imaging outcome measures. J. Cognitive Neurosci. 20, 20-35.

Griffeth, V. E., Perthen, J. E., Buxton, R. B., 2011. Prospects for quantitative fMRI: investigating the effects of caffeine on baseline oxygen metabolism and the response to a visual stimulus in humans. Neuroimage 57, 809-816.

Heatherley, S. V., Hayward, R. C., Seers, H. E., Rogers, P. J., 2005. Cognitive and psychomotor performance, mood and pressor effects of caffeine after 4, 6 and $8 \mathrm{~h}$ caffeine abstinence. Psychopharmacology 178, 461-470.

Heatherley, S. V., 2011. Caffeine withdrawal, sleepiness, and driving performance: what does the research really tell us? Nutr. Neurosci. 14, 89-95.

Herz, R. S., 1999. Caffeine effects on mood and memory. Behav. Res. Ther. 37, 869-879.

Iannetti, G. D., Wise, R. G., 2007. BOLD functional MRI in disease and pharmacological studies: room for improvement? Magn. Reson. Imaging 25, 978-988.

Jacobson, K. A., von Lubitz, D. K., Daly, J. W., Fredholm, B. B., 1996. Adenosine receptor ligands: differences with acute versus chronic treatment. Trends Pharmacol. Sci. 17, 108-113.

James, J. E., 1994. Does caffeine enhance or merely restore degraded psychomotor performance? Neuropsychobiology 30, 124-125.

Kennedy, D. O., Haskell, C. F., 2011. Cerebral blood flow and behavioural effects of caffeine in habitual and non-habitual consumers of caffeine: a near infrared spectroscopy study. Biol. Psychol. 86, 298-306.

Kirsch, I., Weixel, L. J., 1988. Double-blind versus deceptive administration of a placebo. Behav. Neurosci. 102, 319-323.

Koppelstaetter, F., Poeppel, T. D., Siedentopf, C. M., Ischebeck, A., Kolbitsch, C., Mottaghy, F. M., Felber, S. R., Jaschke, W. R., Krause, B. J., 2010. Caffeine and cognition in functional magnetic resonance imaging. J. Alzheimers Dis., 20 Suppl 1, S71-84.

Koppelstaetter, F., Poeppel, T. D., Siedentopf, C. M., Ischebeck, A., Verius, M., Haala, I., Mottaghy, F. M., Rhomberg, P., Golaszewski, S., Gotwald, T., Lorenz, I. H., Kolbitsch, C., Felber, S., Krause, B. J., 2008. Does caffeine modulate verbal working memory processes? An fMRI study. Neuroimage 39, 492-499.

Laurienti, P. J., Field, A. S., Burdette, J. H., Maldjian, J. A., Yen, Y. F., Moody, D. M., 2002. Dietary caffeine consumption modulates fMRI measures. Neuroimage 17, 751-757.

Laurienti, P. J., Field, A. S., Burdette, J. H., Maldjian, J. A., Yen, Y. F., Moody, D. M., 2003. Relationship between caffeine-induced changes in resting cerebral perfusion and blood oxygenation level-dependent signal. Am. J. Neuroradiol. 24, 1607-1611.

Liau, J., Perthen, J. E., Liu, T. T., 2008. Caffeine reduces the activation extent and contrast-tonoise ratio of the functional cerebral blood flow response but not the BOLD response. Neuroimage 42, 296-305.

Liguori, A., Hughes, J. R., Grass, J. A., 1997. Absorption and subjective effects of caffeine from coffee, cola and capsules. Pharmacol. Biochem. Be. 58, 721-726.

Liu, T. T., Behzadi, Y., Restom, K., Uludag, K., Lu, K., Buracas, G. T., Dubowitz, D. J., Buxton, R. B., 2004. Caffeine alters the temporal dynamics of the visual BOLD response. Neuroimage 23, 1402-1413.

Loke, W. H., 1993. Caffeine and automaticity in encoding prelexical tasks: theory and some data. Human Psychopharmacol. 8, 77-95.

Magkos, F., Kavouras, S. A., 2005. Caffeine use in sports, pharmacokinetics in man, and cellular mechanisms of action. Crit. Rev. Food Sci. Nutr. 45, 535-562.

Mednick, S. C., Cai, D. J., Kanady, J., Drummond, S. P., 2008. Comparing the benefits of caffeine, naps and placebo on verbal, motor and perceptual memory. Behav. Brain. Res. 193, 7986. 
Miezin, F. M., Maccotta, L., Ollinger, J. M., Petersen, S. E., Buckner, R. L., 2000. Characterizing the hemodynamic response: effects of presentation rate, sampling procedure, and the possibility of ordering brain activity based on relative timing. Neuroimage 11, 735-759.

Mulderink, T. A., Gitelman, D. R., Mesulam, M. M., Parrish, T. B., 2002. On the use of caffeine as a contrast booster for BOLD fMRI studies. Neuroimage 15, 37-44.

Myerson, J., Emery, L., White, D. A., Hale, S., 2003. Effects of age, domain, and processing demands on memory span: Evidence for differential decline. Aging Neuropsychol. C. 10, 20-27.

Nehlig, A., 1999. Are we dependent upon coffee and caffeine? A review on human and animal data. Neurosci. Biobehav. R. 23, 563-576.

Nehlig, A., 2010. Is caffeine a cognitive enhancer? J. Alzheimers Dis. 20 Suppl 1, S85-94.

Newton, R., Broughton, L. J., Lind, M. J., Morrison, P. J., Rogers, H. J., Bradbrook, I. D., 1981. Plasma and salivary pharmacokinetics of caffeine in man. Eur. J. Clin. Pharmacol. 21, 4552.

Nyberg, L., Dahlin, E., Stigsdotter Neely, A., Backman, L., 2009. Neural correlates of variable working memory load across adult age and skill: dissociative patterns within the frontoparietal network. Scand. J. Psychol. 50, 41-46.

O'Connor, P. J., 2006. Mental energy: Assessing the mood dimension. Nutr. Rev. 64, S7-9.

Ollinger, J. M., Corbetta, M., Shulman, G. L., 2001a. Separating processes within a trial in eventrelated functional MRI. Neuroimage 13, 218-229.

Ollinger, J. M., Shulman, G. L., Corbetta, M., 2001b. Separating processes within a trial in eventrelated functional MRI. Neuroimage 13, 210-217.

Ozsungur, S., Brenner, D., El-Sohemy, A., 2009. Fourteen well-described caffeine withdrawal symptoms factor into three clusters. Psychopharmacology (Berl) 201, 541-548.

Park, D. C., Lautenschlager, G., Hedden, T., Davidson, N. S., Smith, A. D., Smith, P. K., 2002. Models of visuospatial and verbal memory across the adult life span. Psychol. Aging 17, 299-320.

Pelligrino, D. A., Xu, H. L., Vetri, F., 2010. Caffeine and the control of cerebral hemodynamics. J. Alzheimers Dis. 20 Suppl 1, S51-62.

Perera, V., Gross, A. S., Xu, H., McLachlan, A. J., 2011. Pharmacokinetics of caffeine in plasma and saliva, and the influence of caffeine abstinence on CYP1A2 metrics. J. Pharm. Pharmacol. 63, 1161-1168.

Perthen, J. E., Lansing, A. E., Liau, J., Liu, T. T., Buxton, R. B., 2008. Caffeine-induced uncoupling of cerebral blood flow and oxygen metabolism: a calibrated BOLD fMRI study. Neuroimage 40, 237-247.

Portas, C. M., Rees, G., Howseman, A. M., Josephs, O., Turner, R., Frith, C. D., 1998. A specific role for the thalamus in mediating the interaction of attention and arousal in humans. J. Neurosci. 18, 8979-8989.

Rack-Gomer, A. L., Liau, J., Liu, T. T., 2009. Caffeine reduces resting-state BOLD functional connectivity in the motor cortex. Neuroimage 46, 56-63.

Ralevic, V., Burnstock, G., 1998. Receptors for purines and pyrimidines. Pharmacol. Rev. 50, 413492.

Salthouse, T. A., 1988. Resource-reduction interpretations of cognitive aging. Dev. Rev. 8, 238272.

Schiff, N. D., 2008. Central thalamic contributions to arousal regulation and neurological disorders of consciousness. Ann. NY Acad. Sci. 1129, 105-118.

Serences, J. T., 2004. A comparison of methods for characterizing the event-related BOLD timeseries in rapid fMRI. Neuroimage 21, 1690-1700.

Snel, J., Lorist, M., Tiegers, Z., 2004. Coffee, Caffeine, and Cognitive Performance. In: Nehlig, A., (Ed), Coffee, Tea, Chocolate, and the Brain. CRC Press, Florida, pp. 53-72.

Song, X. W., Dong, Z. Y., Long, X. Y., Li, S. F., Zuo, X. N., Zhu, C. Z., He, Y., Yan, C. G., Zang, Y. F., 2011. REST: a toolkit for resting-state functional magnetic resonance imaging data processing. Plos One 6, e25031.

Sousa, V. C., Assaife-Lopes, N., Ribeiro, J. A., Pratt, J. A., Brett, R. R., Sebastiao, A. M., 2011. Regulation of hippocampal cannabinoid CB1 receptor actions by adenosine A1 receptors 
and chronic caffeine administration: implications for the effects of Delta9tetrahydrocannabinol on spatial memory. Neuropsychopharmacol. 36, 472-487.

Suzuki, Y., Uematsu, T., Mizuno, A., Fujii, K., Nakashima, M., 1989. Determination of caffeine in saliva by high-performance liquid chromatography: new sampling method for saliva using filter paper. Ther. Drug Monit. 11, 88-92.

Toro, R., Fox, P. T., Paus, T., 2008. Functional Coactivation Map of the Human Brain. Cereb. Cortex 18, 2553-2559.

Wald, F. D. M., Mellenbergh, G. J., 1990. The short version of the Dutch version of the Profile of Mood States. Nederlands Tijdschrift voor de Psychologie 45, 86-90.

Wendte, R., Snel, J., Tieges, Z., 2003. Misclassificatie van cafeïnegebruik: oorzaken en voorbeelden. Fac. Psychologie, Programmagroep Psychonomie. Universiteit van Amsterdam, Amsterdam, pp. 1-20.

Zylber-Katz, E., Granit, L., Levy, M., 1984. Relationship between caffeine concentrations in plasma and saliva. Clin. Pharmacol. Ther. 36, 133-137. 


\section{Supplementary Material}

The Salivette tube contains a swab that participants placed in their mouth for 2 min. Samples were frozen at $-80^{\circ} \mathrm{C}$ until analysed for caffeine content. Caffeine concentration was determined by capillary zone electrophoresis (CZE) using theophilline as an internal standard. Saliva was extracted from Salivettes by centrifugation for $10 \mathrm{~min}$ at $3000 \mathrm{~g}$. $100 \mu \mathrm{l}$ of saliva was mixed with $100 \mu \mathrm{l}$ of potassium phosphate buffer (100mM, pH 7.4) with $10 \mu \mathrm{g} / \mathrm{mL}$ theophylline. Hereafter, $500 \mu \mathrm{L}$ dichloromethane with propanol-2 (4:1) was added and mixed for $30 \mathrm{~s}$ on a Vortex mixer. Organic phase was separated from the aqueous phase by centrifugation at $1000 \mathrm{~g}$ for $5 \mathrm{~min} .300 \mu \mathrm{L}$ of the organic phase was evaporated and solved in $30 \mu \mathrm{L}$ CZE running buffer (50mM glycine/50 mM SDS, pH 10.5). Samples were run on CZE using caffeine in water as a standard. 